\title{
Surrogate-based multi-objective optimization of a composite laminate with curvilinear fibers
}

\author{
Mahdi Arian Nik, Kazem Fayazbakhsh, Damiano Pasini*, Larry Lessard \\ Department of Mechanical Engineering, McGill University, Macdonald Engineering Building, 817 Sherbrooke West, \\ Montreal, QC, Canada H3A 2 K6 \\ *Corresponding author: damiano.pasini@mcgill.ca; Tel: (+1) 514-398-6295; fax: (+1) 514-398-7365
}

\begin{abstract}
A variable stiffness design can increase the structural performance of a composite plate and provides flexibility for trade-offs between structural properties. In this paper, we examine the simultaneous optimization of stiffness and buckling load of a composite laminate plate with curvilinear fiber paths. The problem, which falls in the area of multi-objective optimization, is formulated and solved through a surrogate-based optimization algorithm capable of finding the set of optimum Pareto solutions. We integrate surrogate modelling into an evolutionary algorithm to reduce the high computational cost required to solve the optimization process. The results show that a curvilinear fiber path can increase both buckling load and stiffness simultaneously over the quasi-isotropic laminate. Furthermore, the optimum direction for varying the fiber angle is dependent on the loading direction and boundary conditions. The results for a plate under uniform compression with free transverse edges shows that varying the fiber orientation perpendicular to the loading direction can increase the buckling load by $116 \%$ with respect to that of a quasi-isotropic laminate.
\end{abstract}

Keywords: variable stiffness; curvilinear fiber; laminate design; optimization. 


\section{Introduction}

The design of a laminated composite structure requires the selection of the constituent materials and their best arrangement within the structure. To this end, traditional and nonconventional design strategies have been developed. With the former, the fiber angle and the thickness within each layer are kept constant and the design involves the search for the optimum stacking sequence that results in the best mechanical properties of the composite structure. With the latter, the fibers are allowed to follow curvilinear paths within the plane of the laminates, thereby giving the freedom to tailor the properties in directions that are favorable to carry the load within the laminate [1].

Variable stiffness laminates can be manufactured using an Automated Fiber Placement (AFP) technique [2]. AFP is capable of combining tape placement and filament winding techniques to overcome the limitations and exploit the benefits of the two manufacturing methods. Tape placement is generally more efficient for manufacturing large flat plates and its use is limited to components with simple geometry. Filament winding has its limitations in terms of the structural geometries that can be produced, which are basically restricted to convex nearly cylindrical shapes. An AFP machine typically has a self-contained fiber placement head with three rotational degrees-of-freedom (DOF), which is then mounted on a motion base with several translational DOF. A mandrel with an additional rotational DOF provides a tool surface on which the tows are placed [3].

The improvement in buckling and in-plane stiffness of variable stiffness over constant stiffness design has been demonstrated by several authors. Hyer and Lee [4] improved the buckling resistance of a plate with a circular hole using a curvilinear fiber path. Lund et al. [5] examined two benchmark problems, i.e. a single-layer and a 16-layer simply supported plate, and concluded that the buckling load can be increased by up to $44 \%$ using a variable stiffness compared to a constant stiffness design. Minimum compliance design for a cantilever beam and a square plate was investigated by Setoodeh et al. [6]. It was found that by allowing the in-plane stiffness to vary spatially, the compliance can be improved up to $36 \%$ and $45 \%$ for a cantilever beam and a square plate, respectively. In a follow up work [7], it was pointed out that variable stiffness design can increase the buckling load of a single-layer and a balanced symmetric $[ \pm \theta]_{\text {s }}$ square plate up to $166 \%$ and $67 \%$, respectively. IJsselmuiden et al. [8] studied different loading 
and boundary conditions on balanced symmetric $[ \pm \theta]_{3 s}$ square plates. Results demonstrated improvements more than $100 \%$ in buckling loads of variable-stiffness compared to the optimum constant stiffness designs. While the above studies are promising because they demonstrate the potential of optimizing the curvilinear fiber paths of composite structures, their approaches did not consider the manufacturing constraints, e.g. the minimum turning radius and the minimum cut length imposed by AFP. As a result, several solutions although theoretically optimum might not satisfy the manufacturing constraints imposed by the AFP machine. To avoid this issue, other researchers redefined the tow steered ply definitions to meet the AFP manufacturing constraints. A continuous function was proposed to describe the fiber path, which can also reduce the number of design variables without compromising structural continuity. Gürdal and Olmedo [9] suggested to model the fiber path with a linear fiber angle variation from one end of a plate to the other. In their work, an analysis for calculating the in-plane stiffness of a plate with the prescribed fiber path was proposed. In another attempt, Olmedo and Gürdal [10] used the same fiber path model and layup to investigate the buckling resistance of the plates under uniform compression. It was found that up to $80 \%$ increase in the buckling load can be achieved compared to constant stiffness laminates. Tatting and Gürdal [11, 12] designed and manufactured flat plates with curvilinear fiber path for maximum buckling load under shear loading. An improvement up to $224 \%$ in the buckling load for variable stiffness compared to a constant stiffness design was found. Alhajahmad et al. [13] used the same fiber path definition and found fiber paths that maximized the buckling load of a plate subjected to pressure and inplane loads. It was shown that the buckling load and the in-plane stiffness are generally unrelated and present opposing design trends. In previous research works [9, 10, 13, 14], curvilinear fiber paths were found to substantially improve one of the properties under investigation while the other property was kept constant. Since the concurrent improvement of the buckling load and inplane stiffness was not examined, the benefits of a variable stiffness design were not fully exploited.

Buckling load and in-plane stiffness are examples of two conflicting objectives that can be simultaneously maximized in the design of composite plates, a problem that falls within the area of multi-objective optimization. Instead of a single optimum solution, this problem has a set of optimum solutions defining the Pareto front, which represents the trade-offs among the objectives [15]. 
Among several design optimization algorithms, evolutionary strategies have been demonstrated to be capable of returning a population of solutions at each iteration of the optimization process. Genetic algorithm (GA), which belongs to this category, has been widely used and recommended for optimizing composite structures [16]. GA, however, being a population-based algorithm, requires a large number of function evaluations to reach the optimum solution. This requisite makes the process of finding an optimum solution computationally expensive. To overcome this issue, the coupling of a surrogate model with GA has been recognized as beneficial. For example, Lee and Lin $[17,18]$ used a linear combination of trigonometric basis functions as a surrogate model to optimize the stacking sequence of a composite laminate. It was claimed that the use of the surrogate model decreased the number of GA iterations by one-third. In another study, Lanzi and Giavotto [19] compared the performance of Radial Basis Function (RBF), Kriging, and Neural Network surrogate modeling techniques to achieve a stiffened composite plate with the minimum weight and maximum buckling load. They concluded that all surrogate models under investigation in their work return similar results for the specific case. In addition, Irisarri et al. [20] used RBF to maximize the buckling and the failure load of a stiffened composite plate. Vandervelde and Milani [21] used a second order polynomial regression as a surrogate model, which was developed by Wang et al. [22], to design and optimize an airplane composite wing free vibration. Surrogate-based optimization has been successfully used also for constant stiffness composite design [17-21]. Surrogate modeling, however, has not been used to optimize variable stiffness composite structures.

The focus of this paper is on maximizing simultaneously in-plane stiffness and buckling resistance of a variable stiffness composite plate using a computationally efficient surrogatebased optimization method. Curvilinear fiber paths are selected such that the optimum result can be manufactured using existing fiber placement machines. In the following sections, the fiber path formulation is first presented before investigating two benchmark cases previously studied in $[9,10,14]$. Analytical expressions for the in-plane and out-of-plane responses of the plate under uniform compression are provided to more accurately calculate the buckling load compared to the aforementioned research. Next, a GA-based optimization technique is briefly described. A discussion of the results follows together with design recommendations and directions for future research. 


\section{Problem Definition}

This section explains the fiber path used to design a variable stiffness plate. Then, two case studies of square plates, each under two dissimilar boundary conditions, are described.

\subsection{Fiber path definition}

For the design of a variable stiffness plate, it is necessary to define a reference path (Figure 1a) along which the AFP machine places the first course (a course is defined as a number of tows placed side by side). The subsequent fiber paths can be obtained by offsetting the machine along a given direction, e.g., $x$ - or $y$-directions.

As suggested by Gürdal and Olmedo [9] a reference fiber path that varies linearly along the $x$-axis of the plate can be formulated as

$$
\theta(x)=\frac{2\left(T_{1}-T_{0}\right)}{a}|x|+T_{0}
$$

Where $\theta$ represents the fiber orientation, $a$ denotes the plate width, $T_{0}$ and $T_{1}$ are the fiber angles at the plate center $(x=0)$ and the plate edge $(x=a / 2)$, respectively. In this case, since the path varies along the $\mathrm{x}$-axis, the reference path should be shifted along the y-direction to manufacture the entire plate (Figure 1b). A single layer with this fiber path definition may be represented by $\left\langle T_{0} \mid T_{1}\right\rangle$, where $T_{0}=T_{1}$ represents a straight fiber case. Similarly (Figure 1c), the fiber orientation can be formulated along the $y$-direction, in which $x$ replaces $y$ in Equation (1); in this case, the AFP machine should be offset along the $x$-direction.

\subsection{Case studies}

A square plate $0.254 \times 0.254 \mathrm{~m}$ and made of 16 -ply balanced symmetric $[ \pm \theta]_{4 s}$ laminate subjected to a uniform end shortening along the $x$-direction is considered as a case study. Concerning the boundary conditions, the transverse edges are considered free in case I-a, while the edges are fixed against displacement in the $y$-direction in case I-b (Figure 2). The plates are 
designed with material properties: $E_{x}=181 \mathrm{GPa}, \quad E_{y}=10.27 \mathrm{GPa}, G_{x y}=7.17 \mathrm{GPa}$ and $v_{x y}=0.28$ and with the curvilinear fiber path given by Equation (1) in two different scenarios: case-I, the fiber orientation varies along $x$-direction (parallel to the loading direction); case-II, the fiber orientation varies along $y$-direction (perpendicular to the loading direction).

\section{Analysis}

In this section, the analytical formulations for evaluating two different objectives, namely inplane stiffness and critical buckling load, for a composite plate with a curvilinear fiber path are derived.

\subsection{In-plane response of a variable stiffness plate}

Closed-form expressions for in-plane stiffness evaluation of a variable stiffness plate can be obtained from the in-plane plate equilibrium equations given by

$$
\begin{aligned}
& N_{x, x}+N_{x y, y}=0 \\
& N_{y, y}+N_{x y, x}=0
\end{aligned}
$$

where $x$ and $y$ are the plate coordinates, $N_{x}, N_{y}$, and $N_{x y}$ are the corresponding stress resultants, and the comma denote the partial derivatives of the term with respect to the subscripts. Employing the generalized stress-strain relations and assuming a linear relation between displacement and strain, the above equations take the form:

$$
\begin{aligned}
& A_{11} u_{, x x}+2 A_{16} u_{, x y}+A_{66} u_{, y y}+A_{16} v_{, x x}+\left(A_{12}+A_{66}\right) v_{, x y}+A_{26} v_{, y y}+\left(A_{11, x}+A_{16, y}\right) u_{, x} \\
& +\left(A_{16, x}+A_{66, y}\right) u_{, y}+\left(A_{16, x}+A_{26, y}\right) v_{, x}+\left(A_{12, x}+A_{26, y}\right) v_{, y}=0 \\
& A_{16} u_{, x x}+\left(A_{12}+A_{66}\right) u_{, x y}+A_{26} u_{, y y}+A_{26} u_{, y y}+A_{66} v_{, x x}+2 A_{26} v_{, x y}+A_{22} v_{, y y} \\
& +\left(A_{16, x}+A_{12, y}\right) u_{, x}+\left(A_{66, x}+A_{26, y}\right) u_{, y}+\left(A_{66, x}+A_{26, y}\right) v_{, x}+\left(A_{26, x}+A_{22, y}\right) v_{, y}=0
\end{aligned}
$$


where $A_{i j}$ are the elements of the in-plane stiffness matrix of the composite plate, $u$ and are $v$ mid-plane displacement in the $x$ - and $y$-direction, respectively. For more details on deriving these equations, interested readers may refer to [14].

Unlike a constant stiffness design, the derivatives of the A matrix with respect to $x$ and $y$ elements are not zero. This difference is a result of varying the fiber path in the xy plane. Since the above two partial differential equations with variable coefficients are coupled, a numeric solutions must be obtained. MATLAB subroutines have been developed to solve the above equations and calculate the displacement distribution of the plate as well as the equivalent inplane stiffness.

\subsection{Buckling response of a square plate}

This section presents the calculation of the critical buckling load of a variable stiffness plate subjected to an in-plane uniform compression (constant displacement along $x$-direction, $u_{0}$ ). The plate is assumed to be simply supported along all edges; these geometrical conditions at the boundaries imply zero out-of-plane deflection along the edges. The compression load at the plate edge $\left(N_{x 0}\right)$, corresponding to the displacement $u_{0}$, is obtained using the equations of the inplane response mentioned in Section 3.1. To calculate the buckling load, $N_{x 0}$ is increased proportionally $\left(\lambda N_{x 0}\right)$ where $(\lambda)$ is the load multiplier. $\lambda_{c r}$ is defined as the minimum load multiplier for a buckled plate and can be obtained using an energy method [23]. The Ritz method is used to obtain the critical buckling load and the out-of-plane deflection $(w)$ of the plate. Since all edges are simply supported, $w$ should be zero along these edges. The following series approximation that satisfies the boundary conditions is assumed for $w$

$$
w=\sum_{i=1}^{I} \sum_{j=1}^{J} C_{i j} \sin \frac{i \pi x}{a} \sin \frac{j \pi y}{a}
$$

where $I$ and $J$ are arbitrary numbers used in the summations, and $a$ is the plate width. $C_{i j}$ are constants which can be calculated using the principle of stationary potential energy defined as 


$$
\frac{\partial \Pi}{\partial C_{i j}}=\frac{\partial(U+\Omega)}{\partial C_{i j}}=0
$$

where $\Pi$ represents total potential energy, $U$ is the strain energy due to the out of plane deflection of the plate and $\Omega$ denotes the potential energy due to the external forces. The strain energy due to the out-of-plane deflection of the plate can be calculated as

$$
\begin{aligned}
U=\frac{1}{2} \int_{0}^{a} \int_{0}^{a}\left[D_{11}\left(\frac{\partial^{2} w}{\partial x^{2}}\right)^{2}+D_{22}\left(\frac{\partial^{2} w}{\partial y^{2}}\right)^{2}+D_{66}\left(\frac{2 \partial^{2} w}{\partial x^{2}}\right)^{2}\right. \\
\left.+2\left(D_{12} \frac{\partial^{4} w}{\partial x^{2} \partial y^{2}}+D_{16} \frac{\partial^{4} w}{\partial x^{3} \partial y}+2 D_{26} \frac{\partial^{4} w}{\partial x \partial y^{3}}\right)\right] d y d x
\end{aligned}
$$

where $U$ represents the strain energy, $D_{i j}$ denotes the elements of the bending stiffness matrix and $w$ is the out-of-plane deflection of the plate. These equations take into account the bending/twisting coupling terms, i.e. $D_{16}$ and $D_{26}$ of the bending stiffness matrix. We note that these terms were neglected by previous researchers to simplify the calculations $[10,14]$. However, neglecting even small $D_{16}$ and $D_{26}$ terms may result in a significant error in the buckling load estimation [24]. The potential energy due to the external force can be obtained by

$$
\Omega=\frac{1}{2} \int_{0}^{b a} \int_{0}^{a}\left[N_{x}\left(\frac{\partial w}{\partial x}\right)^{2}+N_{y}\left(\frac{\partial w}{\partial y}\right)^{2}+2 N_{x y} \frac{\partial^{2} w}{\partial x \partial y}\right] d y d x
$$

Since the material properties, i.e. stiffness and Poisson's ratio, change within the plane due to varying fiber angles, the compression load at the plate edge may not be constant. Thus, $\mathrm{N}_{\mathrm{x}}^{\text {ave }}$ the average compression load (per unit length) along the plate edge, can be defined as

$$
N_{x}^{\text {ave }}=\frac{\int_{-\frac{a}{2}}^{\frac{a}{2}} N_{x}\left(\frac{a}{2}, y\right) d y}{a}
$$

Substituting Eq. (4) into Eqs. (6) and (7) and then replacing into Eq. (5) yields an eigenvalue problem as follows 


$$
\sum_{i=1}^{I} \sum_{j=1}^{J}\left(G_{m n i j}-\lambda b_{m n i j}\right) w_{i j}=0 \quad\left\{\begin{array}{l}
i, m=1,2,3, \ldots, I \\
j, n=1,2,3, \ldots, J
\end{array}\right.
$$

where the elements of $[G]$ are the summation of the integral terms in Eqs. (6) and (7) containing the $D_{i j}$ 's and the elements of [b] are the sums of the integral terms containing $N_{x}, N_{y}$, and $N_{x y}$. The lowest eigenvalue (load multiplier) corresponds to the lowest buckling load [23].

\section{Formulation of the optimization problem}

Variable stiffness design provides flexibility for trade-offs between different structural properties. The involvement of two or more conflicting objectives results in a multi-objective optimization problem. The solution of this problem is not unique; instead of a single optimum solution, there is a set of optimum solutions representing the trade-off between objectives. In this work, buckling resistance and in-plane stiffness of a plate, which are generally conflicting to each other, are considered as objective functions. The goal here is to find the fibre path parameters (design variables) that simultaneously maximize the in-plane stiffness and buckling resistance. One way to convert a maximization problem into a minimization problem is to use the inverse of the objective functions. Then, the optimization problem can be written as follows

$$
\begin{aligned}
& \min _{\mathbf{x}}\left\{1 / E_{e q}(\mathbf{x}), 1 / N_{c r}(\mathbf{x})\right\} ; \mathbf{x}=\left(T_{0}, T_{1}\right)^{T} \\
& \text { s.t. }\left\{T_{0}, T_{1} \in\left[0^{\circ}, 90^{\circ}\right]\right\}
\end{aligned}
$$

Where $\mathbf{x}$ is the vector of design variables, i.e., $T_{0}$ and $T_{1}$ that are the fiber angle at the plate center and plate edge, respectively; $E_{e q}$ and $N_{c r}$ are respectively the equivalent in-plane stiffness and the critical buckling load of the plate. It is worth to mention that the design variables should be integers to respect the manufacturing constraints. To reduce the computational cost involved in the optimization process, we now resort to a surrogate modeling approach which will be coupled with the optimization algorithm. 


\subsection{NSGA-II and motivation for surrogate modeling}

Non-dominated sorting genetic algorithm-II (NSGA-II) is a multi-objective Evolutionary Algorithm (EA) that uses elite-preservation strategy and explicit diversity-preserving mechanism to find a set of evenly distributed solutions to a multi-objective optimization problem [25]. NSGA-II was developed to reduce the computational complexity, to improve the diversity among non-dominated solutions and to add elitism to non-dominated sorting genetic algorithm (NSGA) [26]. Simplicity, effectiveness, and independency on user-defined parameters, make NSGA-II a flexible and robust EA procedure for solving various multi-objective optimization problems using a common framework [27]. As mentioned in the introduction, NSGA-II needs a large number of function evaluations to find the Pareto optimal set. However, for the case of this study since the objective functions are evaluated through expensive simulations, the high computational cost of the multi-objective optimization becomes a major challenge.

For this reason, we resort here to a surrogate model that approximates the computationally expensive simulation through a multi-dimensional parametric surface [28, 29]. The surrogate model, which is significantly cheaper to evaluate than the high fidelity simulation, replaces the expensive simulations during the optimization process; as a result, the computational cost of the optimization process can be significantly reduced.

\subsection{Surrogate modeling}

There are a variety of techniques to construct surrogate models. Since their performance is problem-dependent, the best surrogate model is not known at the outset. We thus emphasize that a comparative study of the performance of various surrogate models should be performed before selecting the best surrogate model for a specific design problem. This task, however, is beyond the scope of this work. In this section, the method of polynomial regression (PR), which is the simplest non-linear model for constructing a surrogate model, is selected and its formulation is briefly described. 


\subsubsection{Polynomial Regression (PR)}

A second-order polynomial can be expressed as

$$
\tilde{f}(\mathrm{x})=\beta_{0}+\sum_{i=1}^{n} \beta_{i} x_{i}+\sum_{i=1}^{n} \beta_{i i} x_{i}^{2}+\sum_{i=1}^{n} \sum_{j=i+1}^{n} \beta_{i j} x_{i} x_{j}
$$

where $\beta_{0}, \beta_{i}, \beta_{i i}$ and $\beta_{i j}(i, j=1, \ldots, n)$ are the regression coefficients, $x_{i}(i=1, \ldots, n)$ are the design variables, and $\tilde{f}$ denotes the approximate value for the objective function. The coefficients of the surrogate model are evaluated through fitting the model to the initial data samples using the least square method [28]. For a number of data samples $\left(N_{s}\right)$ and two design variables $\left(x_{1}\right.$ and $\left.x_{2}\right)$, the set of equations specified in Equation (13) can be written in matrix form as

$$
\mathbf{f}=\boldsymbol{\Phi} \boldsymbol{\beta}
$$

where $\mathbf{f}$ is a $N_{s} \times 1$ matrix of function values at data samples, and $\boldsymbol{\Phi}$ is a $N_{s} \times 6$ matrix, also known as Gramian matrix, of design variable values as the data samples defined by

$$
\boldsymbol{\Phi}=\left[\begin{array}{cccccc}
1 & x_{11} & x_{21} & x_{11} x_{21} & x_{11}^{2} & x_{21}^{2} \\
1 & x_{12} & x_{22} & x_{12} x_{22} & x_{12}^{2} & x_{22}^{2} \\
\vdots & \vdots & \vdots & \vdots & \vdots & \vdots \\
1 & x_{1 i} & x_{2 i} & x_{1 i} x_{2 i} & x_{1 i}^{2} & x_{2 i}^{2} \\
\vdots & \vdots & \vdots & \vdots & \vdots & \vdots \\
1 & x_{1 N_{s}} & x_{2 N_{s}} & x_{1 i} x_{2 N_{s}} & x_{1 N_{s}}^{2} & x_{2 N_{s}}^{2}
\end{array}\right]
$$

The vector $\boldsymbol{\beta}$ of the coefficients of Equation (13) can then be obtained by

$$
\boldsymbol{\beta}=\left(\boldsymbol{\Phi}^{T} \boldsymbol{\Phi}\right)^{-1} \boldsymbol{\Phi}^{T} \mathbf{f}
$$




\subsection{Multi-objective optimization framework}

In the conventional NSGA-II, all individuals in the population are evaluated by the true function, whereas in the surrogate-based NSGA-II only some of the individuals are evaluated by the true functions [30]. The main steps implemented here for a surrogate-based NSGA-II are (Figure 3):

Step 1-The initial sample points are generated by the Latin Hypercube (LH) method [28] . The LH method is used to generate an initial population that is predominantly random, but is uniform in each dimension.

Step 2-Two different surrogate models are constructed based on the initial sample points: the first one predicts the equivalent plate stiffness, and the second one predicts the corresponding buckling load.

Step 3-The intermediate population (offspring) is generated by the standard genetic operators.

Step 4-The individuals in the offspring are divided into two groups (based on their Euclidean distance to the parent individuals): those which lie in the local vicinity of the individuals of the parent population are evaluated by the surrogate model; the remaining individuals (second group) are assessed through true function evaluation.

Step 5-The non-dominate sorting algorithm is applied to create a new population.

Step 6-If any individual in the new population has been evaluated by the surrogate model, then it must be re-evaluated by the true functions before proceeding to the next step.

Step 7-Proceed to step 2 unless the stopping criteria (which requires reach the maximum number of true function evaluation) is satisfied.

\section{Results}

In this section, first the viability of PR surrogate model in increasing the performance of the design optimization of variable stiffness composite structures is verified. In particular, single objective optimization problems, which have known solutions, are examined. Then, the surrogate model is integrated into NSGA-II to solve multi-objective optimization problems. 


\subsection{PR surrogate model performance in single objective optimization problems}

The PR surrogate model is integrated into a conventional GA to maximize the in-plane stiffness of a flat plate with free transverse edges (case II-a). Figure 4 shows the result of the each algorithm, which are averaged over five trials. The result clearly shows superiority of the surrogate-based optimization approach in reducing the computational cost of the optimization compared to the GA.

In a similar approach, the performance of GA and surrogate-based GA in maximizing the buckling load is compared in Figure 5, where the results are averaged over five trials. Also this graph indicates superiority of the surrogate based optimization in reducing the computational cost over the GA.

\subsection{Multi-objective optimization results}

The surrogate-based NSGA-II (NSGA-II+PR) is used to solve the multi-objective optimization problems (Eq. (10)). In each case, the stopping criterion is set to be the maximum number of function evaluations, i.e. 500. The in-plane stiffness and the buckling load of the variable stiffness plate are normalized using the corresponding values for a constant stiffness quasi-isotropic laminate with $[45 / 0 /-45 / 90]_{2 s}$ layup.

The optimization results for a plate with free transverse edges (case I-a and II-a) and fixed transverse edges (case I-b and II-b) are illustrated in Figure 6 and 7, respectively. A wide extent of the Pareto front was captured for each case. A designer can select any solution placed on the Pareto front considering the desirable trade-off between in-plane stiffness and buckling.

\section{Discussion}

Figures 4 and 5 show that PR surrogate-based optimization algorithms perform better than the GA. In stiffness optimization problem, GA reaches the optimum solution after performing 200 function evaluations, whereas surrogate-based GA requires 116 function evaluations only. Buckling load optimization also confirms the dominance of the surrogate-based GA over GA. The solution achieved by the surrogate-based GA after 72 function evaluations is achieved by 
GA after 225 function evaluations. Therefore, it can be concluded that in this design problem PR surrogate model significantly increases the efficiency of the optimization process.

Figure 6 shows the Pareto fronts obtained with surrogate-based NSGA-II for a flat plate maximizing both in-plane stiffness and buckling load and subjected to free transverse edges. According to classical lamination theory, the obvious solution for maximum stiffness is $[0]_{16 T}$ laminate with all fibers aligned along the loading direction $(x)$. By comparing case I-a and II-a it can be observed that the direction of the fiber orientation angle variation does not play a major role in the maximum achievement in stiffness, i.e. $160 \%$ higher compared to the quasi-isotropic laminate. However, the direction is important for buckling load maximization. It should be recalled that for case $\mathrm{I}-\mathrm{a}$ the fiber orientation changes along the loading direction $(\theta=\theta(x))$, whereas for case II-a the fiber orientation varies perpendicular to the loading direction $(\theta=\theta(y)$ ). In case II-a, an improvement of $116 \%$ in the buckling load can be obtained for $[ \pm<90 \mid 15>]_{4 s}$ laminate at the cost of $46 \%$ reduction in the stiffness over the quasi-isotropic laminate. On the other hand, case I-a can only achieve $20 \%$ improvement in the buckling load for $[ \pm 45]_{4 s}$ laminate along with $62 \%$ reduction in stiffness. For case I-a and II-a, there is a set of optimum solutions in which both stiffness and buckling load are higher compared to the quasi-isotropic laminate (Figure 6). Case II-a generally provides a better set of non-dominated solutions compared to case I-a. Thus, plates with varying fiber orientation perpendicular to the loading direction $(\theta=\theta(y))$ can provide a better performance for stiffness and buckling load compared to the case where the fiber orientation changes along the loading direction.

Figure 7 shows the Pareto fronts for a flat plate with fixed transverse edges. Similar to the previous cases, $[0]_{16 T}$ gives the maximum improvement in stiffness. For case I-b, the fiber orientation changes along the loading direction $(\theta=\theta(x))$, whereas for case II-b the fiber orientation varies perpendicular to the loading direction $(\theta=\theta(y))$. In case II-b, at the extreme value of the buckling load, an improvement of $37 \%$ is achieved for $[ \pm<90 \mid 10>]_{4 s}$ laminate which comes at the cost of $14 \%$ reduction in stiffness over the quasi-isotropic laminate. On the other hand, in case I-b for $[ \pm<0 \mid 49>]_{45}$ laminate both buckling load and stiffness can be increased by $32 \%$ and $51 \%$, respectively. Unlike the case with free transverse edges (case I-a and II-a), most of the solutions on the Pareto fronts have both higher stiffness and buckling load 
compared to the quasi-isotropic laminate. Case I-b gives a significantly better set of solutions compared to case II-b. Therefore, square plates with varying fiber orientation parallel to the loading direction can provide an improved performance for stiffness and buckling load compared to the case where the fiber orientation changes perpendicular to the loading direction.

\section{Conclusion and recommendations}

This paper has examined the multi-objective optimization of the in-plane stiffness and buckling load of a composite flat plate with variable stiffness. Surrogate-based GA and GA have been compared for two single objective optimization problems, i.e. stiffness and buckling load maximization. The surrogate-based optimization has been demonstrated to perform better than GA in solving the optimization problem of variable stiffness composite plate.

For a flat plate with free transverse edges, varying fiber orientation perpendicular to the loading direction can improve the buckling load by $116 \%$ over the quasi-isotropic laminate. On the other hand, for a flat plate with fixed transverse edges, the maximum achievable improvement in the buckling load is only $37 \%$. For all cases, using curvilinear fiber path results in a set of optimum solutions in which both stiffness and buckling load are higher than a quasiisotropic laminate. For example, $[ \pm<0 \mid 49>]_{45}$ laminate can increase both buckling load and stiffness over quasi-isotropic laminate by $32 \%$ and $51 \%$, respectively. Hence, the optimum direction of varying the fiber angle is dependent on the loading and boundary conditions. For free edges, the optimum direction is perpendicular to the loading direction while for fixed transverse edges it is along the loading direction.

Alternative fiber paths, e.g. constant curvature fiber path, might bring additional improvement in the simultaneous optimization of stiffness and buckling load. Further work, however, is required to investigate this matter. In addition, a future work will compare the performance of alternative surrogate models to identify the one that can most efficiently reduce the computational cost required to solve the multi-objective optimization of composite laminate structures with curvilinear fibers. 


\section{Acknowledgement}

This work was supported by the National Research Council of Canada, Bombardier Aerospace and Composites Atlantic.

\section{References}

[1] Ghiasi H, Fayazbakhsh K, Pasini D, Lessard L. Optimum stacking sequence design of composite materials Part II: Variable stiffness design. Composite Structures. 2010;93:1-13.

[2] Evans DO, Vaniglia MM, Hopkins PC. Fiber placement process study. 34th International SAMPE Symposium and Exhibition. Reno, Nevada; USA;1989. p. 1822-33.

[3] Gürdal Z, Tatting BF, Wu KC. Tow-placement technology and fabrication issues for laminated composite structures. 46th AIAA/ASME/ASCE/AHS/ASC Structures, Structural Dynamics \& Materials Conference. Austin, Texas, 2005. p. 1-17.

[4] Hyer M, Lee H. The use of curvilinear fiber format to improve buckling resistance of composite plates with central circular holes. Composite Structures. 1991;18:239-61.

[5] Lund E, Stegmann J. On structural optimization of composite shell structures using a discrete constitutive parametrization. Wind Energy. 2005;8:109-24.

[6] Setoodeh S, Abdalla MM, Gürdal Z. Design of variable-stiffness laminates using lamination parameters. Composites Part B: Engineering. 2006;37:301-9.

[7] Setoodeh S, Abdalla M, Ijsselmuiden S, Gürdal Z. Design of variable-stiffness composite panels for maximum buckling load. Composite Structures. 2009;87:109-17.

[8] Ijsselmuiden ST, Abdalla MM, Gürdal Z. Optimization of Variable-Stiffness Panels for Maximum Buckling Load Using Lamination Parameters. AIAA journal. 2010;48:134-43.

[9] Gürdal Z, Olmedo R. In-plane response of laminates with spatially varying fiber orientations: variable stiffness concept. AIAA journal. 1993;31:751-8.

[10] Olmedo R, Güdal Z. Buckling Response of Laminates With Spatially Varying Fiber Orientations. American Institute of Aeronautics and Astronautics, S. W, Washington, D. C. 20024-2518, USA; 1993. p. 2261-9.

[11] Tatting BF, Gürdal Z. Design and manufacture of elastically tailored tow placed plates. NASA contractor report no NASA/CR-2002-211919. 2002.

[12] Tatting BF, Gürdal Z. Automated finite element analysis of elastically-tailored plates. NASA contractor report no NASA/CR-2003-212679. 2003.

[13] Alhajahmad A AM, Gürdal Z. Optimal Design of Tow-Placed Fuselage Panels for Maximum Strength with Buckling Considerations. Journal of Aircraft. 2010;47:775-82.

[14] Gürdal Z, Tatting BF, Wu CK. Variable stiffness composite panels: Effects of stiffness variation on the in-plane and buckling response. Composites Part A: Applied Science and Manufacturing. 2008;39:911-22.

[15] Deb K. Multi-objective optimization using evolutionary algorithms: Wiley, 2001.

[16] Ghiasi H, Pasini D, Lessard L. Optimum stacking sequence design of composite materials Part I: constant stiffness design. Composite Structures. 2009;90:1-11.

[17] Lee Y-J, Lin C-C. Regression of the response surface of laminated composite structures. Composite Structures. 2003;62:91-105.

[18] Lin C-C, Lee Y-J. Stacking sequence optimization of laminated composite structures using genetic algorithm with local improvement. Composite Structures. 2004;63:339-45.

[19] Lanzi L, Giavotto V. Post-buckling optimization of composite stiffened panels: Computations and experiments. Composite Structures. 2006;73:208-20.

[20] Irisarri FX, Laurin F, Leroy FH, Maire JF. Computational strategy for multiobjective optimization of composite stiffened panels. Composite Structures. 2011;93:1158-67. 
[21] Vandervelde T, Milani AS. Layout optimization of a multi-zoned, multi-layered composite wing under free vibration. Proceedings of SPIE, the International Society for Optical Engineering San Diego, CA, USA 2009.

[22] Wang GG, Dong Z, Aitchison P. Adaptive response surface method - A global optimization scheme for approximation-based design problems. Engineering Optimization. 2001;33:707-33.

[23] Kollar LP, Springer GS. Mechanics of composite structures: Cambridge University Press, 2003.

[24] Turvey GJ. Buckling and postbuckling of composite plates: Springer, 1995.

[25] Deb K, Pratap A, Agarwal S, Meyarivan T. A fast and elitist multiobjective genetic algorithm: NSGA-II. Evolutionary Computation, IEEE Transactions on. 2002;6:182-97.

[26] Srinivas N, Deb K. Muiltiobjective optimization using nondominated sorting in genetic algorithms. Evolutionary computation. 1994;2:221-48.

[27] Deb K. A robust evolutionary framework for multi-objective optimization. ACM; 2008. p. 633-40.

[28] Forrester A, Keane A. Recent advances in surrogate-based optimization. Progress in Aerospace Sciences. 2009;45:50-79.

[29] Wang GG, Shan S. Review of Metamodeling Techniques in Support of Engineering Design Optimization. Journal of Mechanical Design. 2007;129:370-80.

[30] Li M, Li G, Azarm S. A Kriging Metamodel Assisted Multi-Objective Genetic Algorithm for Design Optimization. Journal of Mechanical Design. 2008;130:031401-10. 


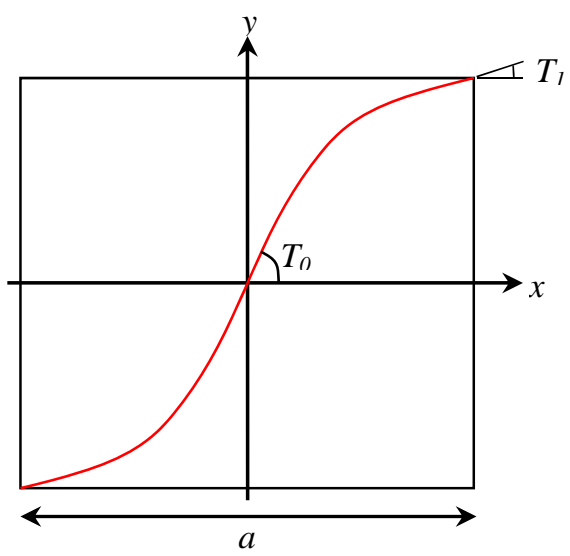

(a)

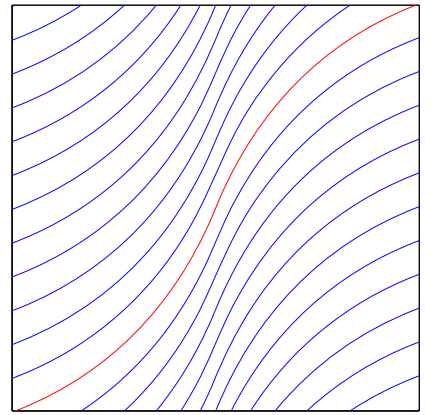

(b)

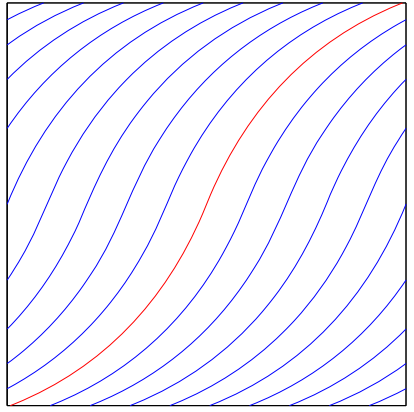

(c)

Figure 1: A composite laminate with a curvilinear fiber path; (a) definition of a reference fiber path; (b) fiber angle offset along $y$-direction $\left(T_{0}=60^{\circ}, T_{1}=15^{\circ}\right)$; (c) fiber angle offset along $x$-direction $\left(T_{0}=60^{\circ}, T_{1}=15^{\circ}\right)$;

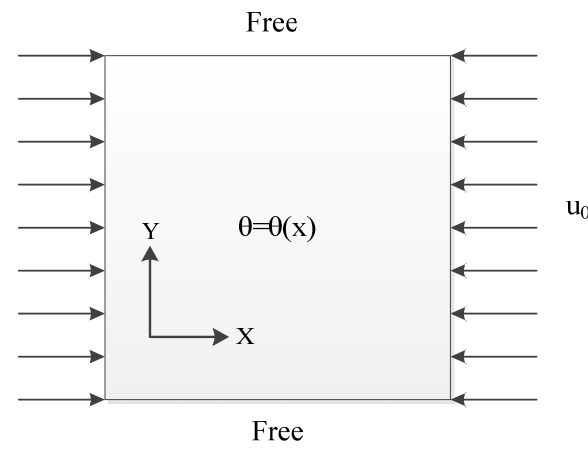

Case I-a: Square panel under uniform compression, Free transverse edges $\mathrm{v}=0$

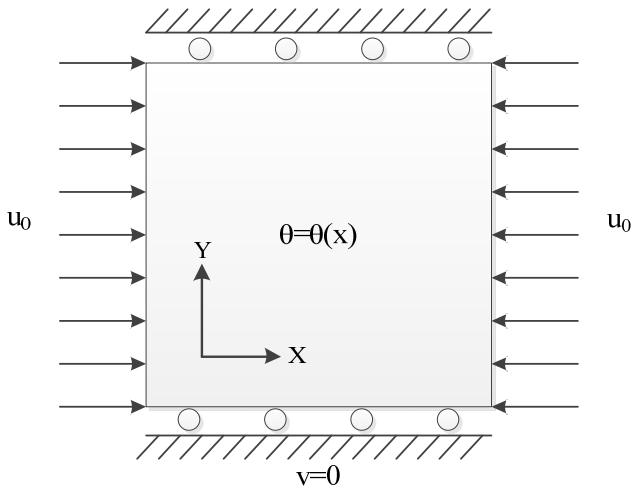

Case I-b: Square panel under uniform compression, Fixed transverse edges

Figure 2. Case I: Square plates with different boundary conditions. 


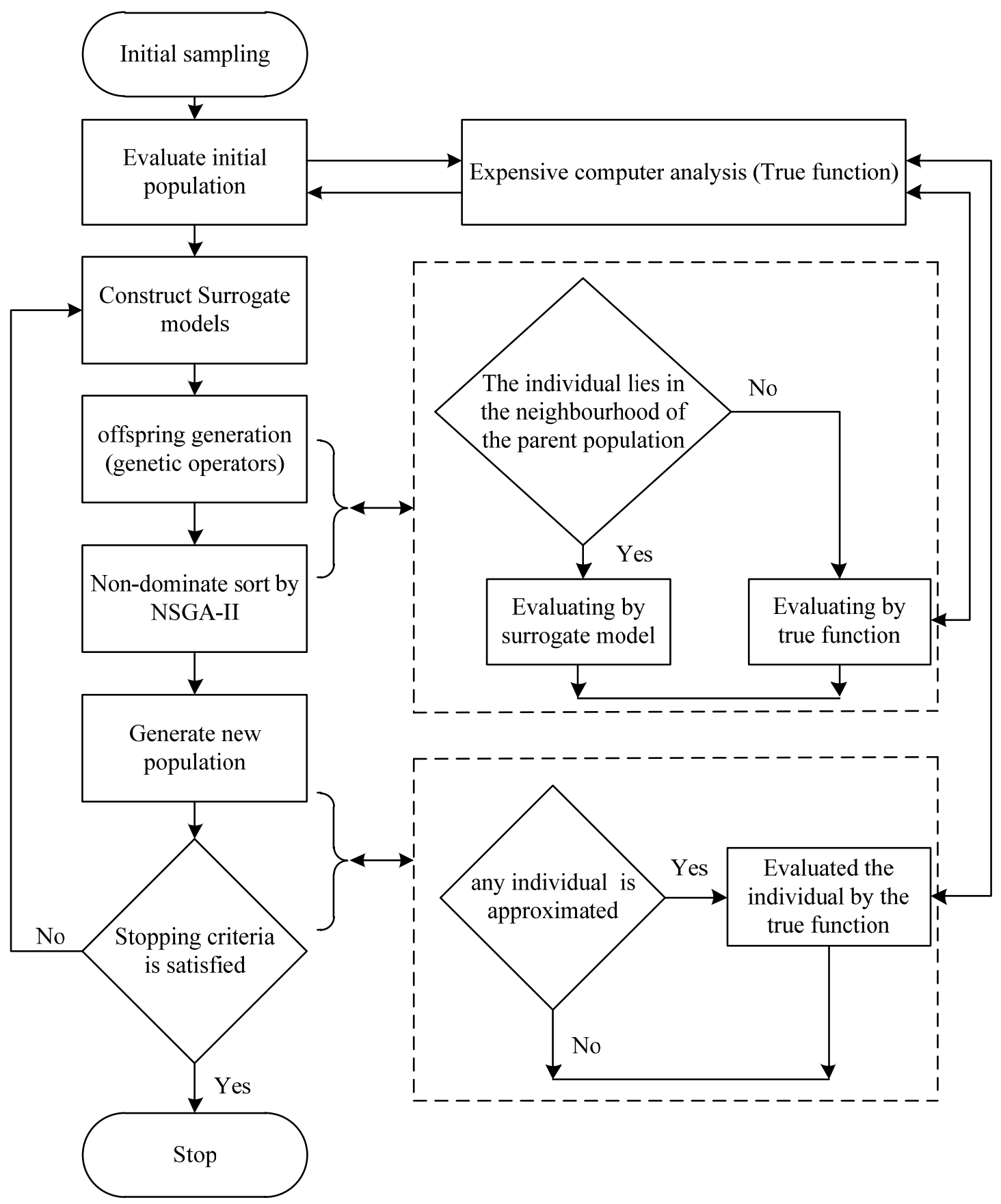

Figure 3. Flowchart of surrogate-based optimization. 


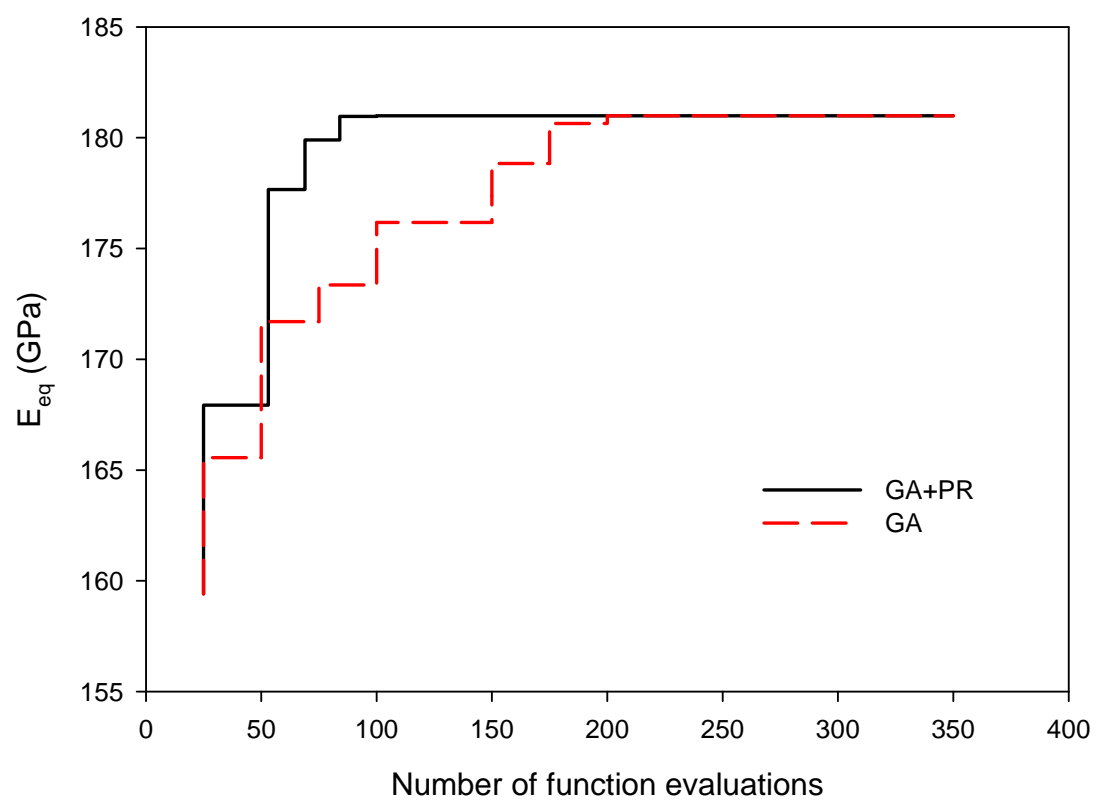

Figure 4. Performance comparison of surrogated based optimization. The results are averaged over 5 trials.

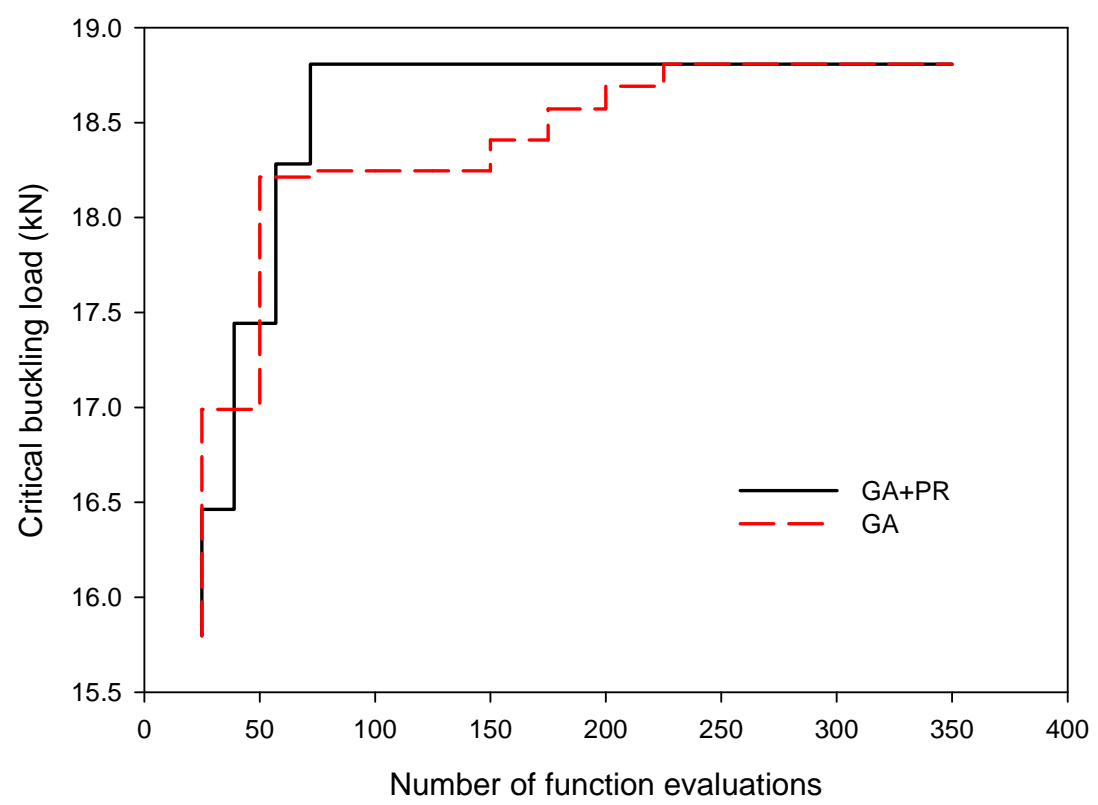

Figure 5. Performance evaluation of surrogated based optimization. The results are averaged over 5 trials. 


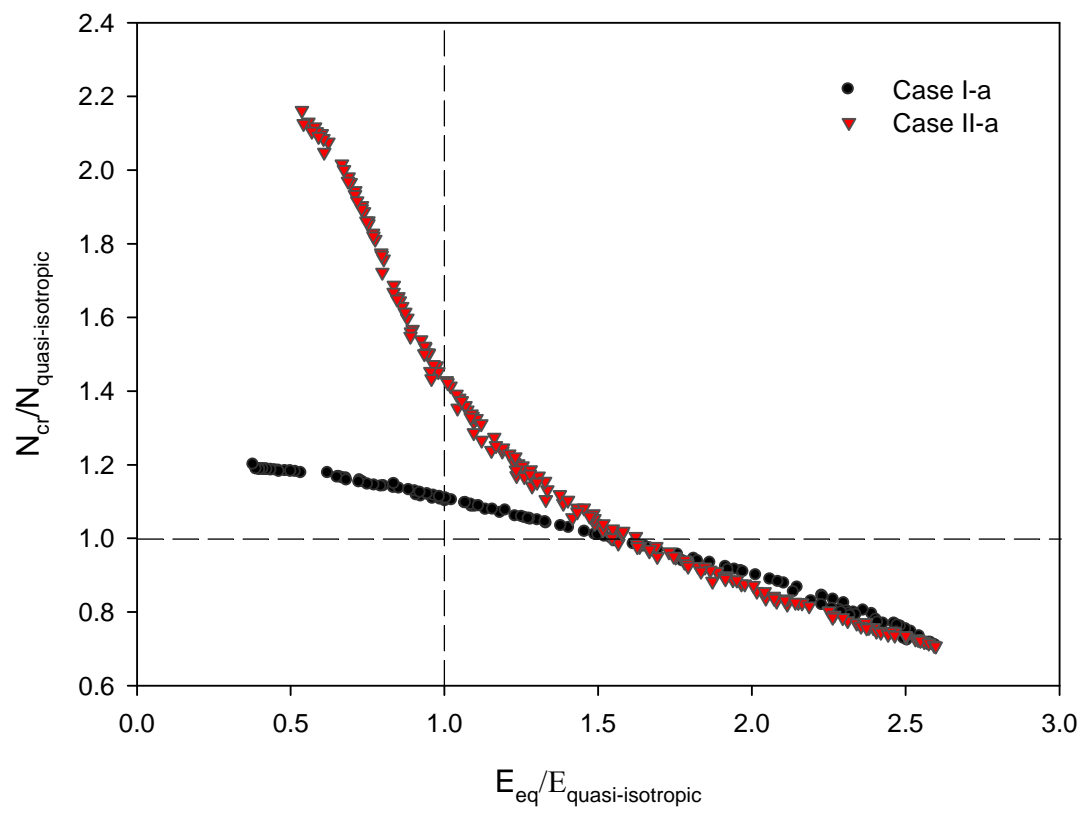

Figure 6. Pareto set obtained by maximizing the buckling load and the overall plate stiffness.

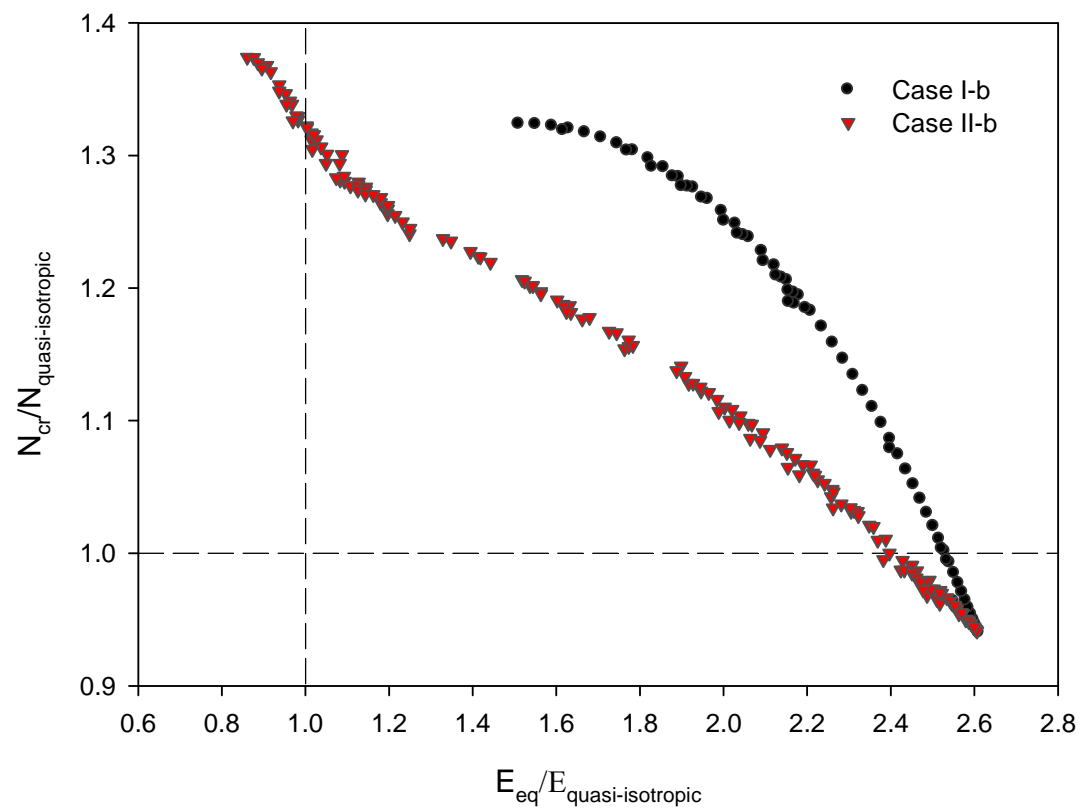

Figure 7. Pareto set obtained by maximizing the buckling load and the overall plate stiffness. 